Benjamin Kickhöfer, Julia Kern

\title{
Pricing local emission exposure of road traffic: An agent-based approach
}

Journal article | Accepted manuscript (Postprint)

This version is available at https://doi.org/10.14279/depositonce-10068

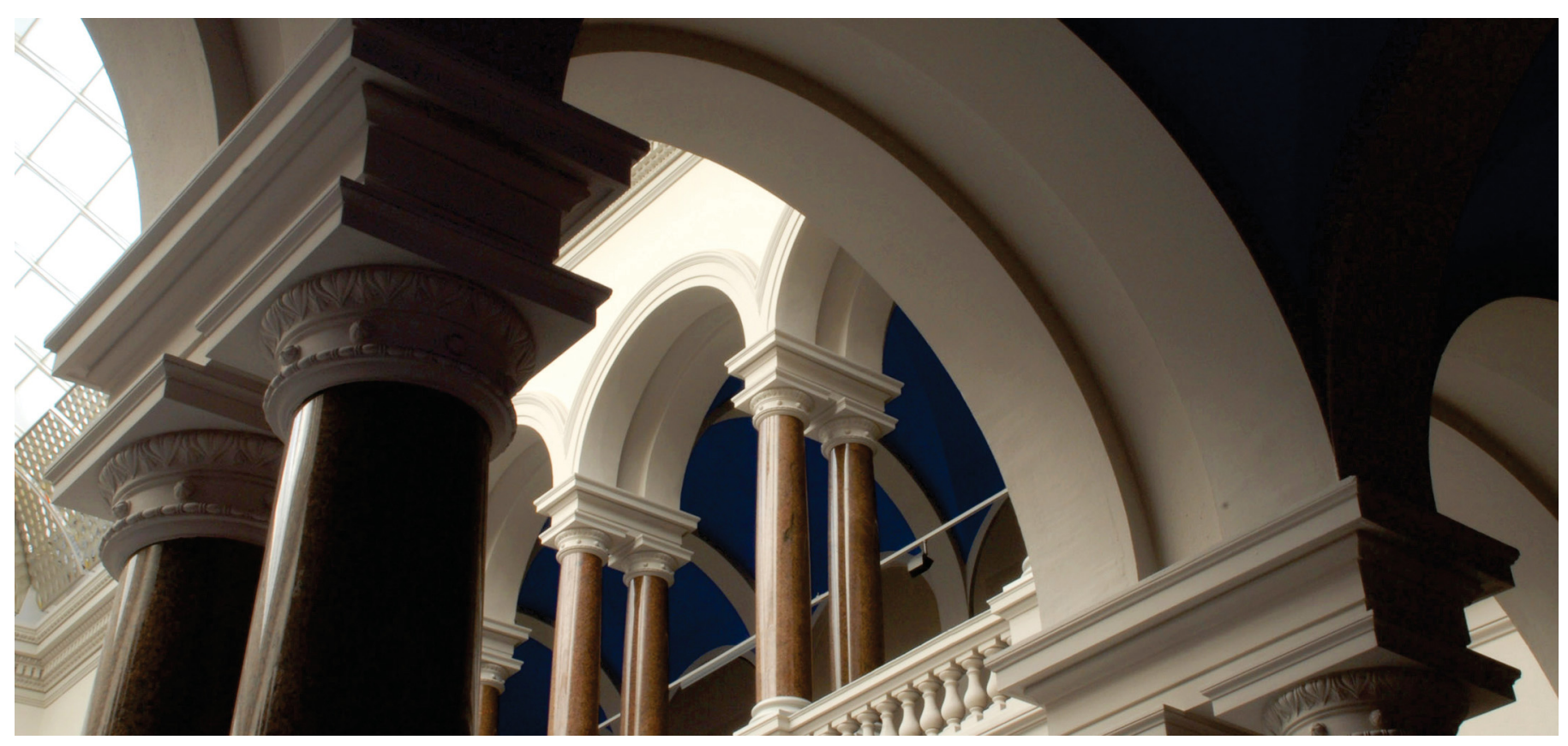

Kickhöfer, B., \& Kern, J. (2015). Pricing local emission exposure of road traffic: An agent-based approach. Transportation Research Part D: Transport and Environment, 37, 14-28. https://doi.org/10.1016/j.trd.2015.04.019 


\title{
Pricing local emission exposure of road traffic: An agent-based approach
}

\author{
Benjamin Kickhöfer, Julia Kern \\ Transport Systems Planning and Transport Telematics Group, Technische Universität Berlin \\ Correspondence address: kickhoefer@vsp.tu-berlin.de
}

July 18, 2014

\begin{abstract}
This paper proposes a new approach to calculate local air pollution exposure costs in large-scale urban settings by taking the number of exposed agents into consideration. It avoids the need for detailed air pollution concentration calculations and is characterized by little data requirements, reasonable computation times for iterative calculations, and open-source compatibility. The approach is applied to a simple test scenario and then to a large-scale real-world scenario of the Munich metropolitan area in Germany. The paper shows (i) how to derive time-dependent vehicle-specific exposure tolls which approximate marginal social costs, (ii) how to estimate changes in system welfare for such pricing scheme when including exhaust emission cost reductions, and (iii) how this approach can be used as benchmark for other transport policy interventions.
\end{abstract}

\section{Introduction}

\subsection{Problem statement}

Negative externalities in the transport sector are known to lead to market inefficiencies and social welfare losses. The latter exist since individuals base their decisions on marginal private and not on marginal social costs, typically yielding demand levels beyond the economic optimum. To correct for these market failures, Pigou (1920) proposed to internalize the difference between marginal social costs and generalized prices by a toll. Since then, the concept has been studied widely in the transportation economic literature (see, e.g., Lindsey and Verhoef, 2000; Small and Verhoef, 2007; Vickrey, 1969; Arnott et al., 1993; Friesz et al., 2004). However, all these studies focus on congestion costs. Other important contributions to the total external costs are found to be air pollution, accidents, and noise (Maibach et al., 2008; Parry and Small, 2005). Since these environmental externalities 
have gained more attention over the last decades (OECD, 2006), and some studies find their impact for some regions at the same level as congestion costs (see, e.g., Creutzig and He, 2009), a new approach was proposed by Kickhöfer and Nagel (2013) to calculate vehicle-specific time-dependent air pollution tolls that reflect marginal emission costs with respect to congestion and vehicle attributes. However, their tolls did not account for population exposure; this drawback will be tackled in the present paper.

Other possibilities to correct for these market failures are discussed in the literature, e.g. so-called backcasting approaches (Geurs and van Wee, 2004; IWW et al., 1998). The idea is to define threshold values based on medical studies, and then to derive avoidance costs in order to reach the desired values. The advantage is that avoidance costs are relatively easy to estimate. However, the definition of threshold values remains rather unclear, and exposure or concentration-response relationships could potentially provide more realistic information since they provide damage cost estimates (WHO Europe, 2006). For the European Union (Holland et al., 2005; Hurley et al., 2005) and the US (U.S. EPA, 2011), this exposure approach typically consists of five steps:

- Modeling exhaust emissions

- Modeling emission dispersion

- Deriving air pollution concentration

- Estimating exposure of individuals to air pollutants with respect to special population groups like pregnant or ill persons, children and elderly

- Applying concentration-response functions yielding numbers of cases for mortality, life years lost, hospital admissions, premature mortality, minor restricted activity days, work loss days, etc.

- Assigning monetary values to each of these cases

\subsection{Emission dispersion and air quality models}

In the literature, a large number of microscopic and macroscopic dispersion models exists. However, as a review paper by Holmes and Morawska (2006) shows, the latter can not provide the spatial resolution that is needed for air pollution concentration modeling within urban-scale scenarios. The former are generally characterized by long computing times and are therefore often not applicable to large-scale urban regions. According to Holmes and Morawska (2006), most emission dispersion and air quality modeling tools need geographical and meteorological input data like temperature, altitude, humidity, cloud cover, peak sun, sunrise, terrain elevation data, land cover data, hourly meteorological data, sea and land breezes. These data might not be available for the area of interest.

Despite these data requirements, there exist several attempts to model air quality in urban regions. Hatzopoulou and Miller (2010) use the open-source modeling tool CALPUFFCALMET to evaluate air quality. Calculation of concentration values for 15'000 areas and 62'500 receptors from link-wise aggregated exhaust emissions initially takes them 190 hours of computing time. Hence, such approach would simply not be manageable for the iterative calculation of toll levels as it is attempted in the present paper. The Community Multiscale Air Quality model (CMAQ) and EPA's Modeled Attainment Test Software 
used by U.S. EPA (2011) have their focus on North American scenarios. When applying the models to European scenarios, Appel et al. (2012) find Particular Matter (PM) concentration values to be underestimated by $24 \%$ to $65 \%$. Holland et al. (2005) use the Cooperative Programme for Monitoring and Evaluation of the Long-range Transmission of Air Pollutants in Europe (EMEP) combined with Regional Air Pollution Information and Simulation (RAINS) on a $50 \times 50 \mathrm{~km}$ grid. However, both tools focus on macroscopic long-range dispersion over whole countries. Hülsmann et al. (2013) focus on emission dispersion modeling for street canyons using the Operational Street Pollution Model (OSPM) for a small area of their scenario. Despite the model's complexity and relatively large data requirements, it could be used for deriving air pollution concentrations and for calculating time-dependent tolls in order to eliminate emission hotspots in the small research area. Unfortunately, the software is not open-source, and can therefore not be integrated into MATSim (see Sec. 2.1) for iterative toll calculations.

\subsection{Simplified approach}

This paper starts from the idea of pricing damage costs to human health. In order to improve the approach by Kickhöfer and Nagel (2013), it proposes how to calculate local air pollution exposure in large-scale urban settings by taking the number of exposed agents into consideration. Additionally, the approach is characterized by little data requirements, reasonable computation times, and open-source compatibility. It is composed of the three following steps:

1. The MATSim ${ }^{1}-\mathrm{HBEFA}^{2}$ emission modeling tool is used which has been developed by Hülsmann et al. (2011) and further improved by Kickhöfer et al. (2013). The tool links MATSim's dynamic traffic flows to detailed air pollution emission factors of HBEFA.

2. The resulting vehicle-specific time-dependent exhaust emissions on every link of the network are spatially dispersed using a Gaussian distribution function. For each agent in the simulation who performs an activity inside the dispersion radius, marginal pollution concentration and exposure time are mapped back to the causing agents.

3. A monetary value is assigned to each traveler's contribution to the overall emission exposure. This results in an individual toll. Since the monetary value is assumed to be equal for every agent exposed to a certain emission concentration, the resulting toll captures the effect of activity location density: driving through a highly populated area results in a higher toll level than driving through a less populated area. Furthermore, the individual toll level at the same location is changing over time of day, since the simulation keeps track of all agents' activity patterns: driving through a highly populated residential area during day time will result in a lower toll than driving through the same area during evening hours. In an iterative process, travelers learn how to adapt their route and mode choice behavior in the presence of this simulated air pollution exposure toll.

\footnotetext{
1 'Multi-Agent Transport Simulation', see www .matsim.org

2 'Handbook Emission Factors for Road Transport', version 3.1, see www.hbefa.net
} 
After a detailed description of the model in Sec. 2, the implementation is tested in a simple test scenario (Sec. 3.1) and then applied to a large-scale real-world scenario of the Munich metropolitan area (Sec. 3.2). The results of the exposure toll are compared to the impacts of the emission toll proposed by Kickhöfer and Nagel (2013). Subsequently, these results are used to evaluate the efficiency of potential real-world policy, a speed limitation in the inner city to $30 \mathrm{~km} / \mathrm{h}$. The paper ends with a conclusion in Sec. 4.

\section{Model}

This section gives an overview of the model to calculate exhaust emission exposure and the resulting agent-specific time-dependent toll levels. The section starts with a short introduction to the agent-based transport simulation MATSim, followed by a description of the emission modeling tool which calculates vehicle-specific warm and cold-start emissions. The emissions are then converted into monetary terms using average cost factors from Maibach et al. (2008). Subsequently, the exhaust emission cost dispersion model is presented. Finally, the idea of using the activity-based demand model for calculating population exposure to air pollution is described. Since, in the present approach, emission costs and not emissions are dispersed, this last step gives the marginal cost factors, and thus the individual toll levels.

\subsection{MATSim}

In the following, only general ideas about the transport simulation with MATSim are presented. For in-depth information of the simulation framework, please refer to Raney and Nagel (2006). In MATSim, each traveler of the real system is modeled as an individual agent. The approach consists of an iterative loop that is characterized by the following steps:

1. Plans generation: All agents independently generate daily plans from survey data. These plans encode among other things their desired activities during a typical day as well as the transport mode for every intervening trip.

2. Traffic flow simulation: All plans are simultaneously executed in the simulation of the physical environment. In the car traffic flow simulation, agents interact on the roads which are simulated as first-in first-out queues with flow and storage capacity restrictions (Gawron, 1998; Cetin et al., 2003).

3. Evaluating plans: All executed plans are evaluated by a utility function with the following functional form:

$$
V_{p}=\sum_{i=1}^{n}\left(V_{p e r f, i}+V_{t r, i}\right)
$$

where $V_{p}$ is the total utility for a given plan; $n$ is the number of activities; $V_{\text {perf }, i}$ is the (positive) utility earned for performing activity $i$; and $V_{t r, i}$ is the (usually negative) utility earned for traveling during trip $i$. Activities are assumed to wrap around the 24-hours-period, that is, the first and the last activity are stitched together. In consequence, there are as many trips between activities as there are activities. 
4. Learning mechanism: Some agents obtain new plans for the next iteration by modifying copies of existing plans. This modification is done by several strategy modules that correspond to the available choice dimensions. The choice between plans is performed within a multinomial logit model.

The repetition of the iteration cycle coupled with the agent database enables the agents to improve their plans over many iterations. The iteration cycle continues until the system has reached a relaxed state. At this point, there is no quantitative measure of when the system is "relaxed"; the cycle is simply continued until the outcome is stable.

\subsection{Emission Calculation and Monetization}

The emission modeling tool was developed and tested by Hülsmann et al. (2011) and was further improved by Kickhöfer et al. (2013). The tool links MATSim's traffic flows to the HBEFA database, and essentially calculates warm and cold-start emissions for private cars and freight vehicles. The former emissions are emitted when the vehicle's engine is already warmed whereas the latter occur during the warm-up phase. In the present model, warm emissions differ with respect to vehicle characteristics, traffic state, and road type. Cold-start emissions differ with respect to vehicle characteristics, accumulated distance, and parking duration.

In a first step, vehicle characteristics are obtained from survey data and typically comprise vehicle type, age, cubic capacity and fuel type. They are then used for very differentiated emission calculations. Where no detailed vehicle information is available, fleet averages for Germany are used. For the calculation of warm emissions, MATSim traffic dynamics are mapped to two HBEFA traffic states: free flow and stop\&go. In order to identify road types, information from network data is mapped to HBEFA road types, such as motorway, trunk road, distributor road, or tertiary road. For the calculation of cold-start emissions, parking duration and accumulated distance are monitored in the simulation. The handbook then provides emission factors for the most common pollutants differentiated among the characteristics presented above. For the present paper, the following pollutants are relevant: Sulfur Dioxide $\left(\mathrm{SO}_{2}\right)$, Particular Matter $(P M)$, Nitrogen Oxides $\left(N O_{x}\right)$, Non-Methane Hydrocarbons $(N M H C)$, and Carbon Dioxide $\left(C_{2} s\right)$.

In a second step, so-called 'emission events' are generated based on these warm and cold emission factors. The events provide information about person, time, link, and absolute emitted values by emission type. The definition of emission events follows the MATSim framework that uses events for storing disaggregated information as objects in JAVA programming language and as XML in output files. Emission event objects can be accessed during the simulation or generated later on in a post-processing of the standard MATSim events.

External cost factors for the relevant pollutants from above are taken from Maibach et al. (2008) (see Tab. 1). These values are average estimates for urban regions in Germany with a population greater than 500'000. We assume these being correct for the average population density of the respective scenario. This becomes important when calculating the emission exposure toll (see later in Sec. 2.4). 
Table 1: Emission cost factors by emission type (Maibach et al., 2008)

\begin{tabular}{lr}
\hline Emission type & Cost factor [EUR/ton] \\
\hline $\mathrm{CO}_{2}$ & 70 \\
$\mathrm{NMHC}_{\mathrm{H}}$ & $1^{\prime} 700$ \\
$\mathrm{NO}_{x}$ & $9^{\prime} 600$ \\
$\mathrm{PM}_{\mathrm{SO}}$ & $384^{\prime} 500$ \\
$\mathrm{~S}$ & $11^{\prime} 000$ \\
\hline
\end{tabular}

\subsection{Emission (Cost) Dispersion}

For emission dispersion of single point sources and long time intervals, Stern et al. (1984) proposed a model with a simple Gaussian distribution function (plume model, see Eq. 2). For multiple and area sources and for emission concentration calculations in urban areas, those authors suggest the box model, a discretization in grid cells as in Fig. 1 (Stern et al., 1984, Chapter 18). Presumably, both models only work for larger numbers of agents and appropriate time intervals. They cannot simulate the dispersion of a single agent or car realistically.

In this paper, the plume model is combined with the box model. Thus, following the plume model, the emission dispersion is modeled by multiplying the emission cost value of every emission event by

$$
\frac{1}{\sqrt{2 \pi \sigma^{2}}} \exp \left(-\frac{(x)^{2}}{2 \sigma^{2}}\right)
$$

where $x$ is the distance between the emitting car to the locations of the exposed persons. The variance $\sigma$ is set to the cell length. Applying this to the box model, emission costs are distributed from the emission origin which is assumed to be the center of the respective link to the nearby cells in such a way that the factors add up to one. The generated distribution factors from the Gaussian distribution function with a variance of one divided by the cell length give the average cost factor in the receptor cell, depending on the distance between source and receptor cell. To keep computational time within reasonable limits, only cells within a maximal distance of four are considered since the factors for cells further away fall below 0.0001. This yields a discrete distribution of emission costs into 25 cells (see Fig. 1).

\subsection{Population Exposure}

Emission exposure depends on the number of persons experiencing a certain concentration level for a certain period of time. Dispersed emission (cost) levels are, thus, multiplied by a factor which represents the respective local population exposure times. To calculate these required exposure times, the simulation time is split into isochronous one hour time bins. For each of these bins the amount of time spent by the agents is recoded for each grid cell. The result is a three dimensional data structure consisting of time bin, horizontal and vertical position, and the aggregated durations of agent's presence in the cell (in person seconds $[P s])$.

To give an example: Consider an area with 160 times 120 cells and time bins that correspond to full hours. If an agent arrives at a location in cell $(25,32)$ at 8:30 and leaves 


\begin{tabular}{|l|l|l|l|l|l|l|}
\hline $0.000 €$ & $0.000 €$ & $0.000 €$ & $0.002 €$ & $0.000 €$ & $0.000 €$ & $0.000 €$ \\
\hline $0.000 €$ & $0.000 €$ & $0.002 €$ & $0.029 €$ & $0.002 €$ & $0.000 €$ & $0.000 €$ \\
\hline $0.000 €$ & $0.002 €$ & $0.029 €$ & $0.132 €$ & $0.029 €$ & $0.002 €$ & $0.000 €$ \\
\hline $0.002 €$ & $0.029 €$ & $0.132 €$ & $0.216 €$ & $0.132 €$ & $0.029 €$ & $0.002 €$ \\
\hline $0.000 €$ & $0.002 €$ & $0.029 €$ & $0.132 €$ & $0.029 €$ & $0.002 €$ & $0.000 €$ \\
\hline $0.000 €$ & $0.000 €$ & $0.002 €$ & $0.029 €$ & $0.002 €$ & $0.000 €$ & $0.000 €$ \\
\hline $0.000 €$ & $0.000 €$ & $0.000 €$ & $0.002 €$ & $0.000 €$ & $0.000 €$ & $0.000 €$ \\
\hline
\end{tabular}

Figure 1: Distribution of 1 EUR of emission costs emitted in the center cell (in grey). Cells beyond the shown area are assumed to have a distribution factor of 0.0.

at 10:15, then $1800 P s$ are added to the array at $(x=25, y=32$, time $=8: 00-9: 00)$, another $3600 P s$ are added to $(x=25, y=32$, time $=9: 00-10: 00)$, and $900 P s$ are added to $(x=25, y=32$, time $=10: 00-11: 00)$.

In the next step, emission (cost) levels after dispersion in every cell (see Sec. 2.3) are multiplied by the aggregated $P s$ in the corresponding cell. This gives a product of exposure times and emission (cost) levels for each grid cell and each time bin. For illustration purposes, consider $2 \mathrm{~g}$ of $\mathrm{SO}_{2}$ emitted in a cell at 9:15. Also consider that 15 people are present in a neighboring cell from 9:00 to 10:00. This neighboring cell has 15 3600 aggregated $P s$ of time spent from 9:00 to 10:00. Following the dispersion approach from Sec. 2.3, $0.132 \cdot 2 g$ are dispersed to the considered cell with the 15 agents. This yields $15 P \cdot 3600 s \cdot 0.132 \cdot 2 g=14256 g P s$ of experienced exposure.

Please note at this point, that the approach presented in this paper does not distribute emission levels, but already monetized emissions in EUR. ${ }^{3}$ This simplification assumes (i) that all pollutants are dispersed in the same manner, (ii) that the external effects of all pollutants depend on exposure in the same way, and (iii) that the average cost factors from Sec. 2.2 are correct for the average exposure time in the scenario. That is, for the illustration from above and an assumed average exposure time over all cells of $5000 \mathrm{Ps}$ between 9:00 and 10:00, the cell with the 15 agents yields external exposure costs of

$$
\frac{14256 g P s}{5000 P s} \cdot 0.011 \frac{\mathrm{EUR}}{g}=0.00319572 \mathrm{EUR}
$$

Actual exposure times of each cell are calculated for every time bin. The correction factor of $\frac{1}{5000 \mathrm{Ps}}$ scales the actual exposure time of the cell to the average exposure time of all cells, which is calculated in every iteration of the simulation. The ratio between actual exposure times and average exposure time is non-negative and the average of all ratios is adds up to one. This highlights the design of the exposure calculation: it is computed in such way that a uniform distribution of the actual exposure times over all cells would result in equality between the sum of marginal external emission costs and the sum of emissions multiplied by the average emission costs values from Sec. 2.2.

\footnotetext{
${ }^{3}$ This is done because of computational performance reasons.
} 


\section{Experiments}

This section introduces the first two experiments in which the new emission exposure model is applied. First, a test scenario is set up in Sec. 3.1 for calculating marginal exposure tolls, and for verifying the behavioral reactions of agents in the model. In Sec. 3.2, the exposure toll approach is then applied to the Munich metropolitan area in Germany. In Sec. 3.2.2, impacts on absolute emission levels, exposure cost reductions, and system welfare are compared to the effects of the emission toll proposed by Kickhöfer and Nagel (2013) who did not account for population exposure. Finally, in Sec. 3.2.3, the exposure toll is used to evaluate the efficiency of a speed limitation in the inner city of Munich to $30 \mathrm{~km} / \mathrm{h}$.

\subsection{Test Scenario}

The simulation setup consists of one active agent with one plan, a simple network and 20 inactive agents (Fig. 2, blue rectangles). The active agent's plan is to leave the home location $\mathrm{A}$ at 6:00 a.m. to drive to work location B, and then to go back at 2:00 p.m. Typical durations for 'home' are set to $12 h$, and for 'work' to $9 h$, respectively. There is no opening or closing time restriction for either activity. The network allows two different routes from A to B, both described by almost the same length and identical travel times. As all links of the network are one-way roads, the active agent can choose between these two routes for the trip to work, but has no choice for the way back home. The overall trip length amounts to $17 \mathrm{~km}$ when using the northern route and one meter less for the southern route. The area around the southern route is populated by the inactive agents, yielding a toll on that route.

For the first iteration in the simulation, the agent is expected to choose the southern route since it is marginally faster. No exposure costs are considered at this point, and both routes are therefore almost equal in terms of generalized costs. For all following iterations, exposure times for each cell are used to calculate the expected emission costs when proposing new routes to the agent. The active agent is forced to re-route in every iteration and to store the route with the according score in the memory. The score of the executed plan containing the specified route is calculated at the end of the respective iteration, following the standard MATSim scoring function (see Eq. 1) ${ }^{4}$. After ten iterations the agent is only allowed to switch between existing plans for the following ten iterations.

Running the test scenario yields the expected results: The agent generates five plans which include the northern route and another five plans with the southern route. The score of the plans containing the southern route is 23.479 utils, the score of the plans containing the northern route is 23.494 utils. This implies additional exposure emission costs on the southern route of 0.015 utils equivalent to 0.190 EUR. Thus, the agent finally chooses the northern route to go to work. Difference between the scoring of the two routes is caused by the toll from traveling on the link near the passive agents. Passing the respective link generates $0.0276 E U R$ of direct emission costs. These are scaled by the according relative exposure duration and distribution factors. In the particular case with one hour time bins the exposure durations are $3600 P s$ for all cells with passive agents since each stays in its

\footnotetext{
4 The behavioral parameters are the same as used for the Munich scenario (see Sec. 3.2): $\beta_{t r}=$ -0.96 utils $/ h$, and $\beta_{c}=-0.0789942$ utils $/ E U R$, and $\beta_{\text {perf }}=+0.96$ utils $/ h$.
} 


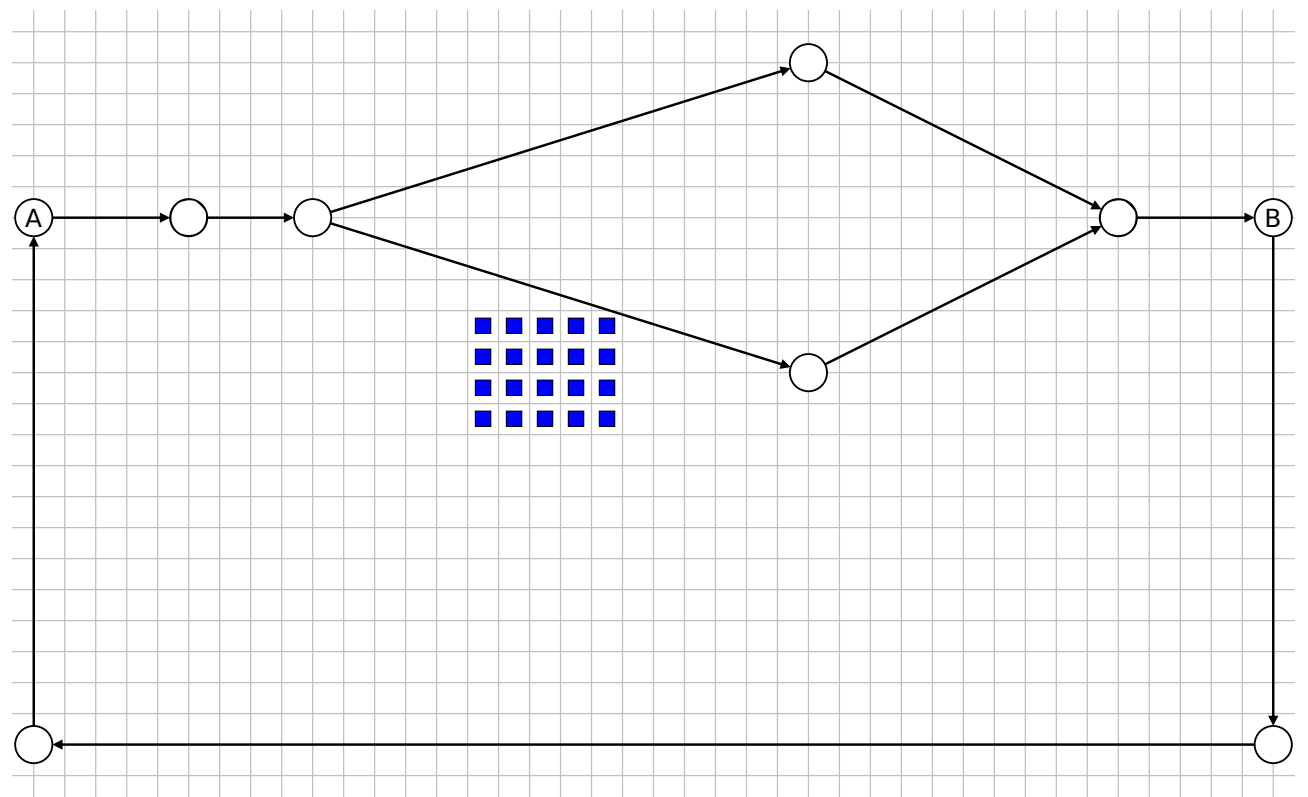

Figure 2: The active agent drives from home location A to work location $\mathrm{B}$ and back to A and choses between two routes. The blue rectangles represent inactive agents. Grey lines mark the boundaries of each cell for exposure calculations.

cell. Consequently the 20 passive agents and 640 cells yield an average exposure duration of approximately $\frac{20 \cdot 3600 P s}{640}=112.5 P s$ per cell. (This is not exact since the active agent itself causes additional exposure durations at its home and work location.)

The generated emissions costs of 0.0276 EUR affect nine populated cells: One with distribution factor 0.132 , three with distribution factor 0.029 and five with distribution factor 0.002 (as explained in Sec. 2.4).

Consequently the imposed toll is

$$
0.0276 \mathrm{EUR} \cdot \frac{(0.132+3 \cdot 0.029+5 \cdot 0.002) \cdot 3600 P s}{112.5 P s}=0.202 \mathrm{EUR}
$$

which is close to 0.190 EUR from the simulation result. The difference can be explained by the additional time the active agent spends at its home and work location leading to a greater average exposure duration and thereby to a lower toll. In consequence, the plausibility test can be regarded as successful: Emission exposure costs influence the decision making of the agent during the simulation.

\subsection{Munich, Germany}

This section now applies the exposure toll to the large-scale real world scenario of the Munich metropolitan area in Germany. It uses the same initial scenario as Kickhöfer and Nagel (2013), and compares impacts on absolute emission levels, exposure cost reductions, and system welfare to the effects of the emission toll (flat toll per gram of pollutant) and of the speed reduction in the inner city that were analyzed in that paper. ${ }^{5}$

\footnotetext{
${ }^{5}$ Please note that the focus of the present paper is on exposure costs which are calculated for both toll schemes. The values in emission cost reductions induced by the two policies from Kickhöfer and Nagel
} 


\subsubsection{Base Case}

In the following the results of the simulation of a no-measure base case equivalent to the setup from Kickhöfer and Nagel (2013) are recapitulated. These results serve as reference case estimations ("business as usual"), against which the flat emission toll and the exposure toll are then compared in the subsequent section. Results are always presented for four subpopulations: urban travelers (who perform trips within the city boundaries of Munich), commuters (who live outside of Munich and travel to the city for work), reverse commuters (who live in Munich and travel outside the city for work), and freight traffic. ${ }^{6}$

As Fig. $3^{7}$ shows, urban travelers contribute to a relatively small part of total emissions despite representing $68 \%$ of the total population which corresponds to approximately 1.3 million individuals. Only $N M H C$ emissions are over-proportionally high. This is due to the fact that urban travelers drive shorter distances, and in general perform more trips, and, in consequence, produce more cold-start emissions. Commuters and reverse commuters ( $14.6 \%$ and $9.8 \%$ of the total population) cause substantially more emissions per person since they drive longer distances. Intuitively, Munich attracts more individuals who additionally come from further away (commuters) than the individuals that are attracted by the surroundings of Munich (reverse commuters). For that reason, the former drive longer distances and emit more emissions per person than the latter. Freight traffic only represents $7.6 \%$ of the total population but causes a major part of total emissions. This is most important for $P M$ and $N O_{x}$. From the initial emission values of the different subpopulations, it already becomes clear who will bear most of the emission toll payments. However, when pricing local emission exposure, this picture is not so clear any more: toll payments will depend on activity location density along the route, and will therefore strongly vary between different trips.

\subsubsection{Emission vs Exposure Toll}

In order to show the effects of the different toll schemes, changes in emission levels by subpopulation are presented first. As can be seen in Fig. 4, the emission toll on the left reduces emission levels of all subpopulations and most pollutants. For example, $N O_{x}$ emissions are reduced by $-0.4 \%$ overall. The effect is most important for urban travelers and least important for freight. Only for reverse commuters and freight, one can observe a slight increase in the cold-start related $N M H C$. In comparison, the exposure toll on the right shows a less homogeneous picture: emission levels are still reduced for urban travelers and commuters, but for reverse commuters and freight, one can even observe an increase in total emission levels for most pollutants. It is, at this point, important to note that the different subpopulations have different options (choice dimensions) to react on the toll schemes. While urban travelers can adjust their route, and can switch to public transit (PT), freight traffic is only allowed to change route. Commuters and reverse commuters have both options available (route and mode choice), but PT is (in the model) generally less attractive for these demand segments because of their long-distance trips. That is, the more substitutes are available for the respective demand segment, the

\footnotetext{
(2013) are therefore different in scale compared to the exposure cost reductions presented here.

${ }^{6}$ For a more detailed description of the demand generation for this scenario, please refer to Kickhöfer et al. (2013).

${ }^{7}$ Please note the different scales for the different pollutants. This scaling is necessary in order to show absolute values of all pollutants in one single graph.
} 


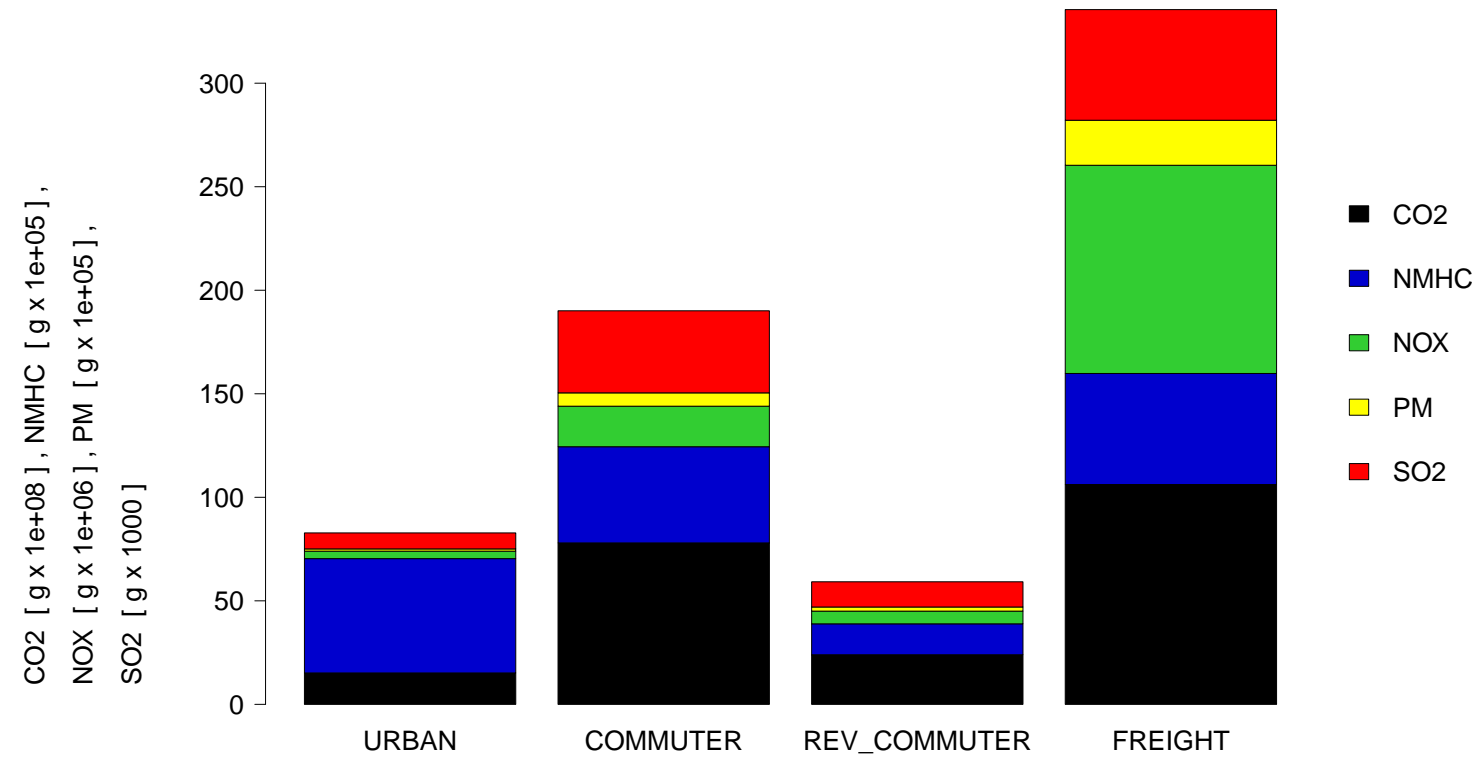

Figure 3: Base case: Absolute emission levels by type and subpopulation. Values scaled to a $100 \%$ scenario.

higher the emission reduction by any of the toll schemes.

Fig. 4 additionally raises the question why the exposure toll increases absolute emission levels for some pollutants (e.g. for $N O_{x}$ by $+0.2 \%$ ). For urban travelers, only $N M H C$ is increasing. This can be explained by the fact that individuals perform more short trips by car where the engine is not completely warmed-up. In addition, less car use leads to cars cooling off for longer periods of time, and therefore to higher cold-start emissions per trip. For reverse commuters and freight traffic, one can observe an interesting particularity of the exposure toll: while routes with less activity locations around them become more attractive, drivers choose longer routes with less potentially exposed individuals, and absolute emission levels therefore increase.

To pick up on this last point, Fig. 5 shows absolute changes in exposure costs by subpopulation. For the exposure toll on the right, substantial reductions in exposure costs can be observed, most important for those caused by freight traffic. That is, even though absolute emissions increase for this demand segment, exposure costs decrease. In contrast, for the emission toll on the left, exposure costs increase even though absolute emission levels drop. This is due to the fact that - with the emission toll - freight vehicles tend to choose shorter routes which might lead through areas with higher activity location density. For the other subpopulations, one can also observe that exposure cost reductions are by a factor of six lower than for the exposure toll. This intuitively makes sense since the emission toll aims for a reduction in absolute emission levels, not for finding the optimal air pollution exposure level.

Tab. 2 and Tab. 3 support the findings from above. They present average toll payments for the whole scenario, and for trips within the boundaries of Munich municipality, respectively. Tab. 2 shows that the average emission toll per car distance traveled for urban travelers, commuters, and reverse commuters ranges from 2.21 to 2.54 EURct $/ \mathrm{km}$. Since freight traffic causes more emissions, the cost factors are consequently in average by a factor of six higher than for the other subpopulations (14.51 EURct $/ \mathrm{km})$. When pricing 


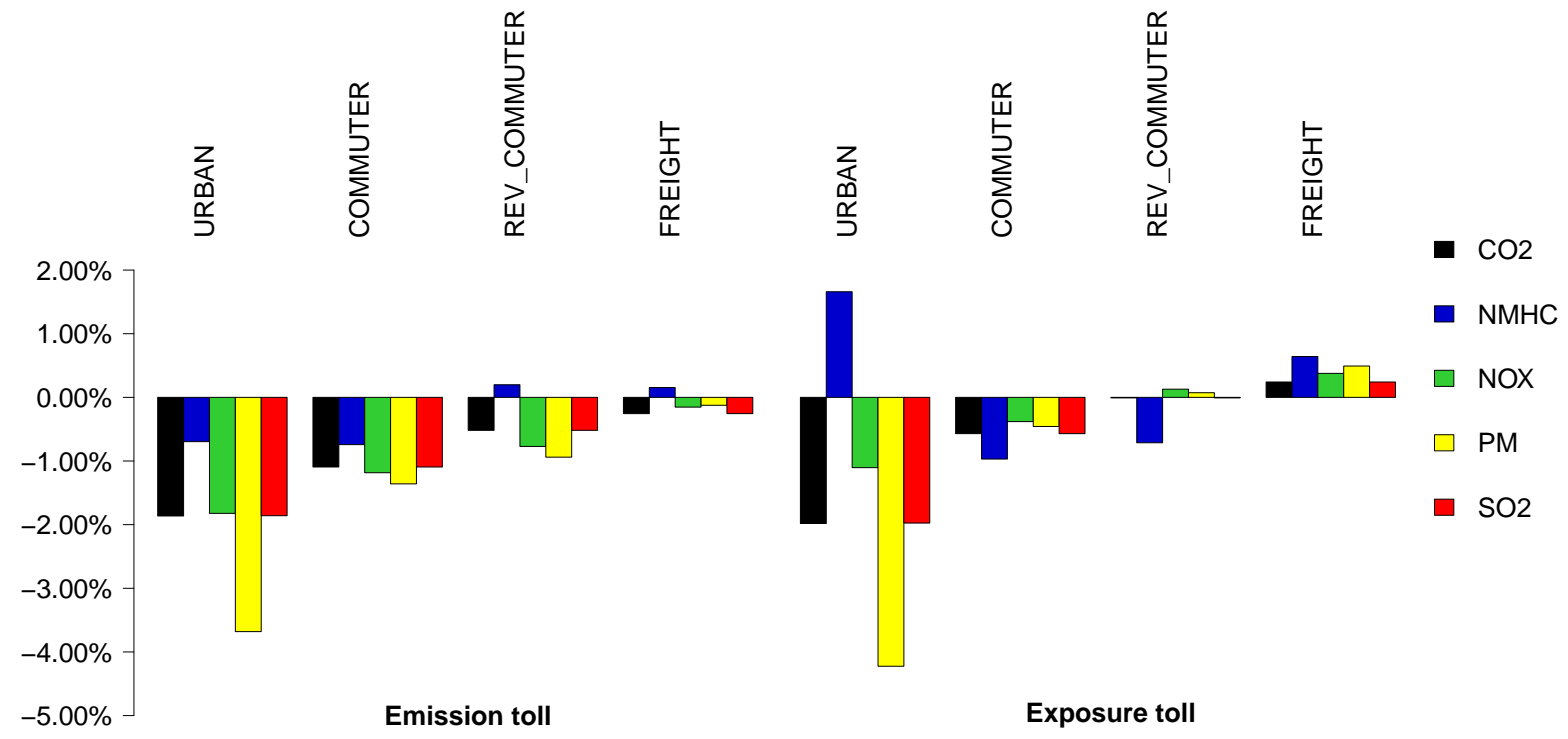

Figure 4: Relative changes in emission levels by subpopulation.

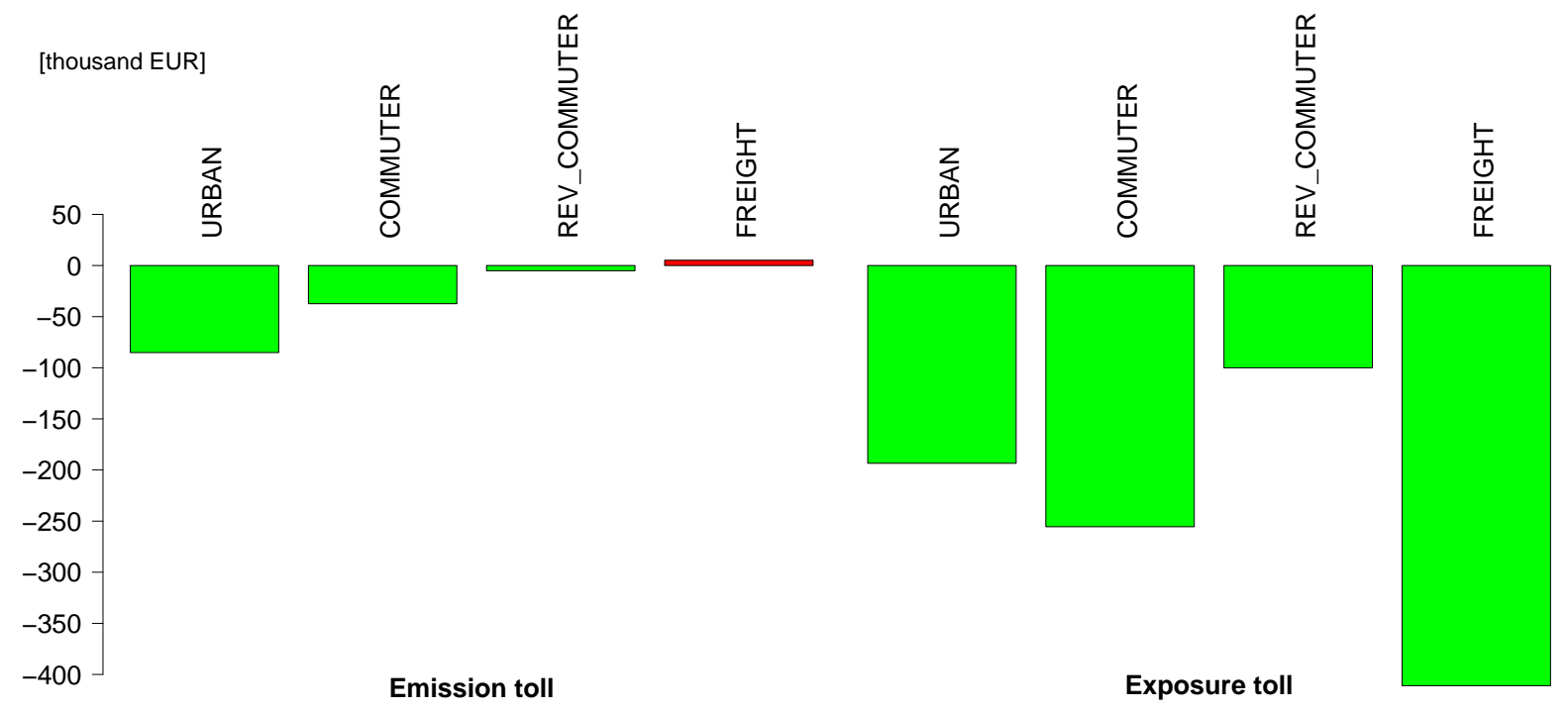

Figure 5: Absolute changes in exposure costs by subpopulation. Values scaled to a 100\% scenario. 


\begin{tabular}{l|l|r|r|r} 
Policy case & Subpopulation & $\begin{array}{r}\text { Total paid } \\
\text { [EUR x 100] }\end{array}$ & $\begin{array}{r}\text { Total car distance } \\
{[\mathrm{km} \text { x 100] }}\end{array}$ & $\begin{array}{r}\text { Average toll } \\
\text { [EURct/km] }\end{array}$ \\
\hline \multirow{3}{*}{ Emission } & URBAN & 1874.58 & 73670.16 & 2.54 \\
Toll & COMMUTER & 9781.59 & 434571.60 & 2.25 \\
& REV. COMM. & 3038.01 & 137568.39 & 2.21 \\
& FREIGHT & 25499.15 & 175769.71 & 14.51 \\
\hline \multirow{3}{*}{ Exposure } & URBAN & 10216.96 & 74488.77 & 13.72 \\
Toll & COMMUTER & 7367.73 & 436856.64 & 1.69 \\
& REV. COMM. & 2315.81 & 138215.81 & 1.68 \\
& FREIGHT & 520.25 & 176685.63 & 0.29
\end{tabular}

Table 2: Average toll payments (overall); values scaled to a $100 \%$ scenario.

\begin{tabular}{l|l|r|r|r} 
Policy case & Subpopulation & $\begin{array}{r}\text { Total paid } \\
{[\text { EUR x 100] }}\end{array}$ & $\begin{array}{r}\text { Total car distance } \\
{[\mathrm{km} \times 100]}\end{array}$ & $\begin{array}{r}\text { Average toll } \\
\text { [EURct/km] }\end{array}$ \\
\hline \multirow{3}{*}{ Emission } & URBAN & 1733.73 & 68164.02 & 2.54 \\
Toll & COMMUTER & 1874.96 & 76784.72 & 2.44 \\
& REV. COMM. & 609.83 & 26220.16 & 2.33 \\
& FREIGHT & 414.99 & 2259.59 & 18.37 \\
\hline \multirow{3}{*}{ Exposure } & URBAN & 9331.85 & 68806.56 & 13.56 \\
Toll & COMMUTER & 6754.35 & 74284.29 & 9.09 \\
& REV. COMM. & 2114.41 & 24903.39 & 8.49 \\
& FREIGHT & 451.93 & 911.85 & 49.56
\end{tabular}

Table 3: Average toll payments (city area); values scaled to a 100\% scenario.

exposure rather than emissions, urban travelers now pay the highest average toll (13.72 EURct $/ \mathrm{km})$, while average tolls of commuters and reverse commuters are roughly 0.50 EURct $/ \mathrm{km}$ lower compared to the emission toll (1.69 and 1.68 EURct $/ \mathrm{km}$, respectively). The average toll for freight now only adds up to 0.29 EURct $/ \mathrm{km}$. Both, the increase in average toll for urban travelers and the decrease for the other subpopulations, can be explained when comparing the car distance traveled from the two tables: While urban travelers drive most of their distance traveled within the boundaries of Munich, where information about activity locations and exposure times is available for this scenario, all other subpopulations only drive a relatively small part of their distance traveled there. In consequence, their average exposure toll levels for the whole scenario are low compared to those of urban travelers. However, when looking at the average exposure toll levels paid in the city area (Tab. 3), one notices a strong increase compared to the overall values. This becomes most important for freight (49.56 EURct $/ \mathrm{km})$.

Another way to show these effects on a more disaggregated level is provided by Fig. 6 . It shows the toll payments for the emission toll (left) and for the exposure toll (right) and all subpopulations as box plots. Data points for these plots are obtained whenever a person leaves a road segment, and therefore has to pay a toll. For every such event, the distance toll payment is divided by the length of that road segment. ${ }^{8}$

For the whole scenario in Fig. 6(a) and the emission pricing scheme, one observes toll

\footnotetext{
8 This means that every road segment has a weight of one in the calculation; the resulting figure therefore implicitly depends on the network topology.
} 

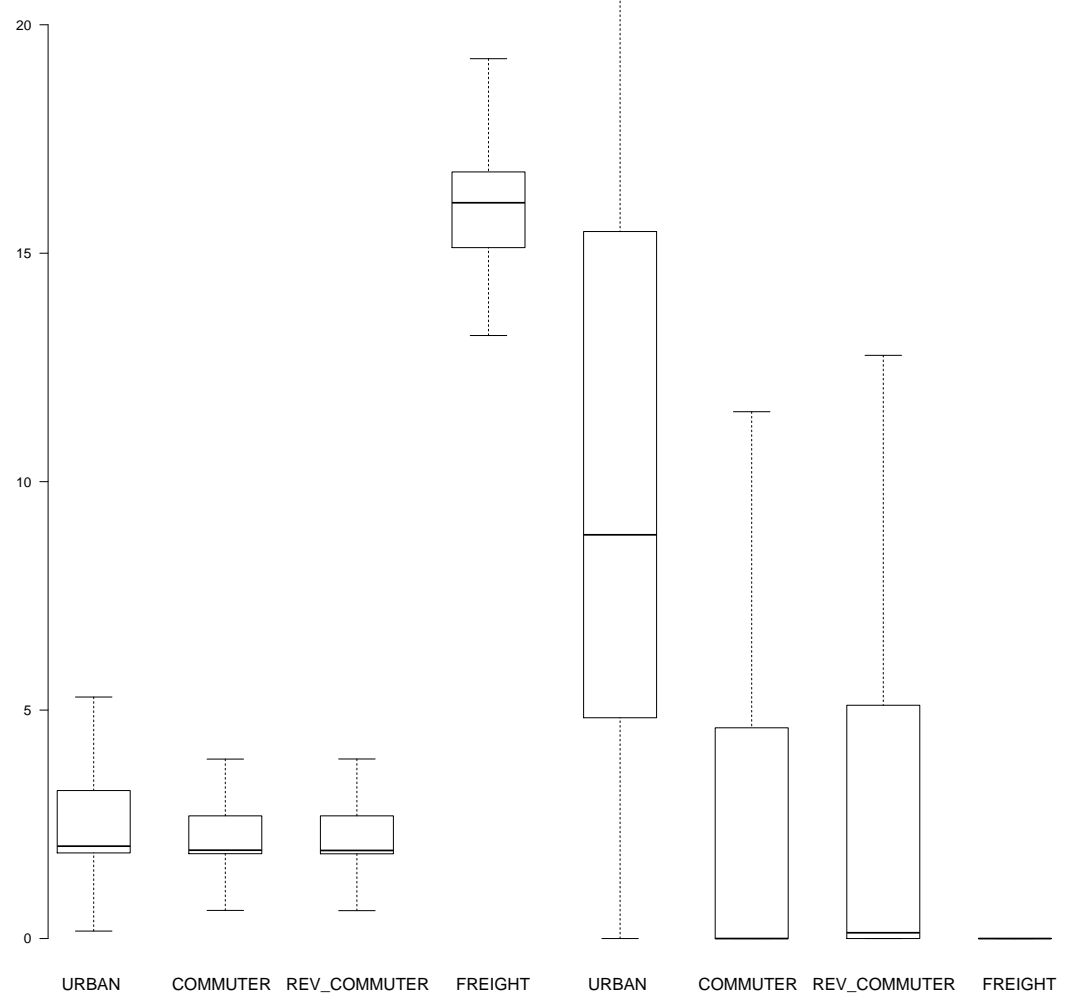

Emission Pricing

Exposure Pricing

(a) Overall
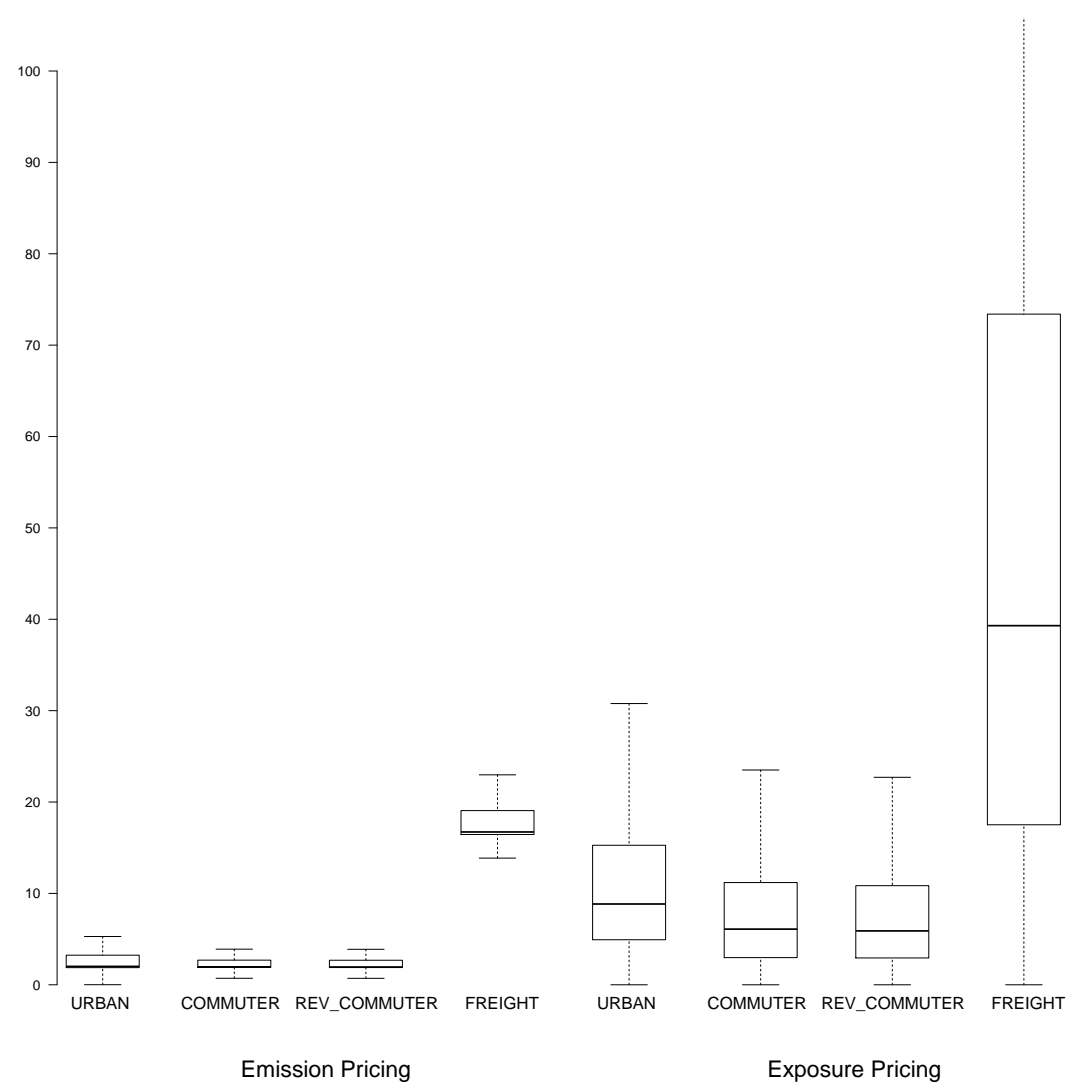

(b) City area

Figure 6: Tolls paid per distance traveled [EURct $/ \mathrm{km}$ ] 
payments around 2.5 EURct/km for urban travelers, commuters, and reverse commuters. The width of the distribution can be explained by different vehicle types, as well as by different traffic states. Both influence emission levels. Since no detailed vehicle types were available for commuters and inverse commuters, the variance can only be explained by different traffic states. The same is true for freight traffic which depicts a rather high average toll. In the same figure, but now for the exposure pricing scheme, the width of the average toll payment distribution strongly increases for the first three subpopulations. This is due to the fact that the toll is now additionally influenced by activity location density (and therefore exposure times) along the route. Only for freight, this effect is not present since this demand segment mostly chooses routes through areas where no activity location information is available in the scenario.

For the tolls paid in the city area of Munich in Fig. 6(b), almost no change is observed for the emission toll (note the scale differences of the $y$-axes). This is consistent with Tab. 2 and Tab. 3. For the exposure toll, the distribution of toll payments for urban travelers remains similar. However, for all other subpopulations, the variance strongly increases, showing the impact of activity location density on the toll level.

\subsubsection{Zone 30 vs Exposure Toll}

In the following, it is discussed how accounting for population exposure in the toll calculations influences the evaluation results of a real-world policy. For that purpose, the results of the exposure toll from Sec. 3.2.2 are compared to a speed limitation in the inner city of Munich to $30 \mathrm{~km} / \mathrm{h}$. This comparison is similar to the one performed by Kickhöfer and Nagel (2013). In contrast to that comparison, the calculation of emission exposure cost reduction now accounts in both policy cases for population exposure: the exposure toll as a benchmark, and the Zone 30 policy as the measure to evaluate.

Fig. 7 shows relative changes in emission levels for all subpopulations. The changes for the exposure toll on the right are identical to the values presented in Fig. 4. For the Zone 30 policy one notices a stronger reduction in emission levels for urban travelers than for the exposure toll. That is, mainly because of mode choice effects, the Zone 30 policy leads to an emission level below the economic optimum. Even though the Zone 30 reduces emission levels of most pollutants for commuters, their and the reverse commuters' emission levels are found to be above the economic optimum. These demand segments choose new routes around the regulated zone; this, in turn, over-compensates the mode switch effect and yields emission levels above the economic optimum. In terms of absolute emissions, the Zone 30 has only a small effect on freight traffic, yielding a emission level below the economic optimum.

Fig. 8 picks up the question where in the city these exhaust emissions are produced and how the resulting monetary changes in exposure costs can be characterized. For urban travelers, the Zone 30 policy seems to be an effective strategy to reduce emission exposure since it yields a similar reduction as the exposure toll (roughly 200 thousand EUR per day). However, as shown in Fig. 7, this reduction in exposure costs requires a stronger reduction in emission levels than the exposure toll, mainly caused by a modal shift towards public transit. For all other subpopulations, the Zone 30 policy leads to an increase in exposure costs, mainly because of re-route effects around the zone. In that sense, the regulatory measure can not be regarded as an efficient strategy to reduce population exposure to exhaust emissions. The presence of this re-route effect is nicely shown in Fig. 9(a). While 


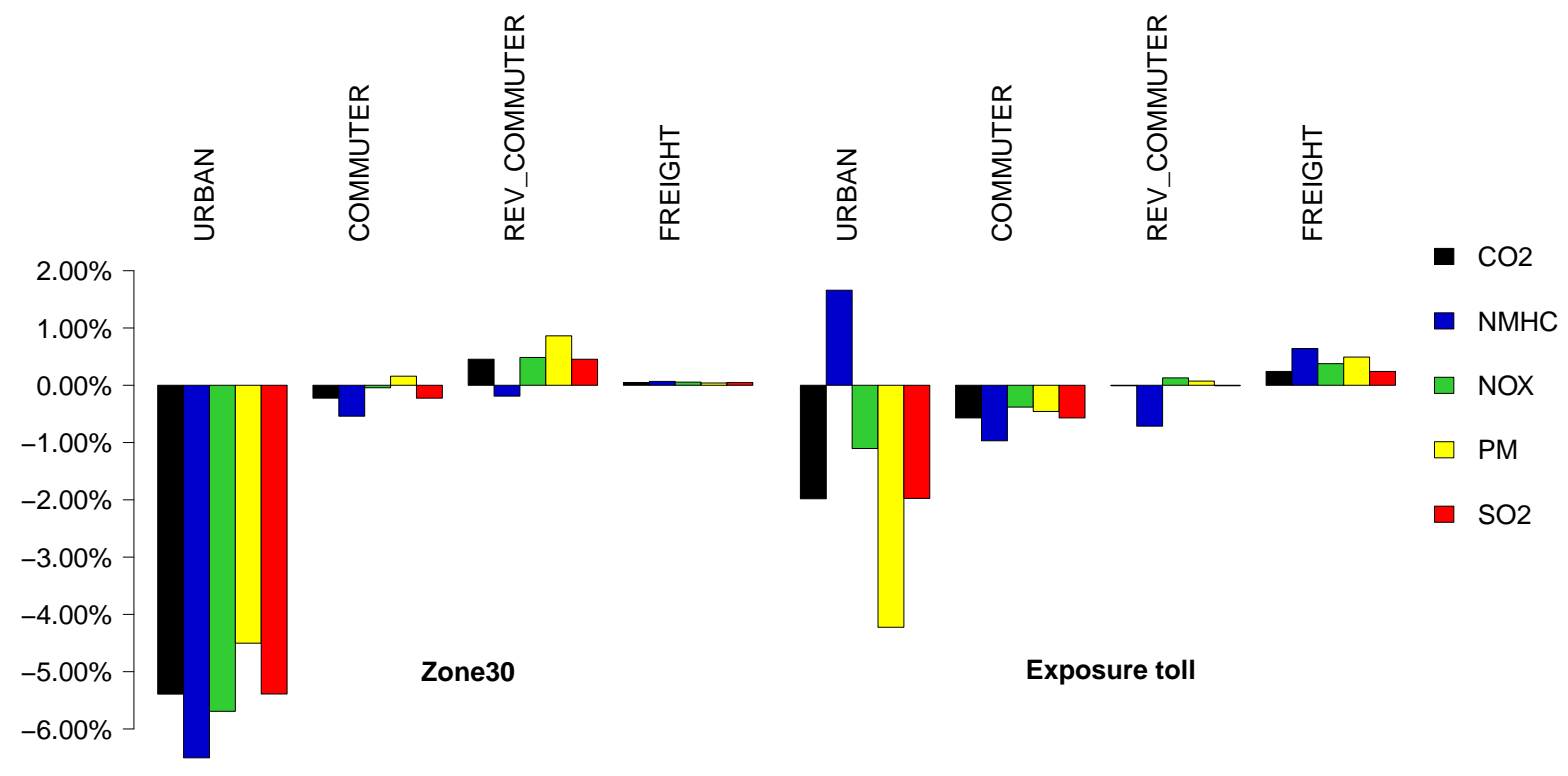

Figure 7: Relative changes in emission levels by subpopulation.

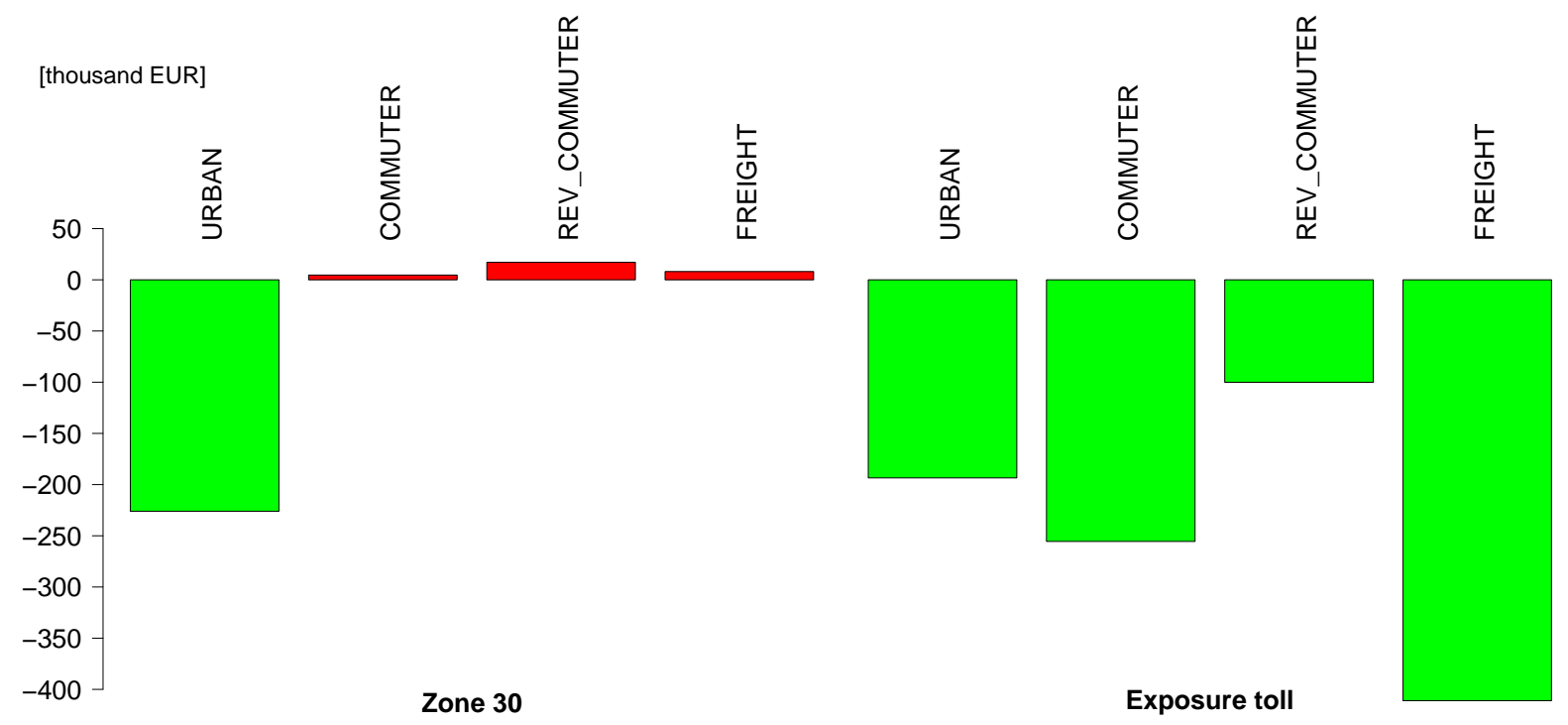

Figure 8: Absolute changes in exposure costs by subpopulation. Values scaled to a $100 \%$ scenario. 


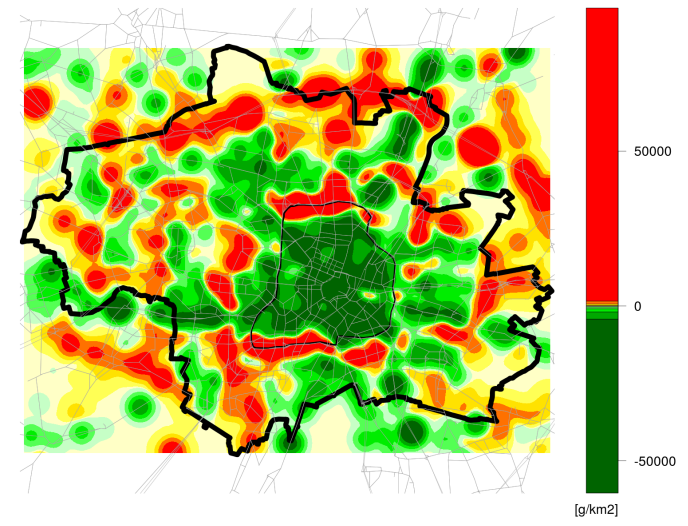

(a) Zone 30

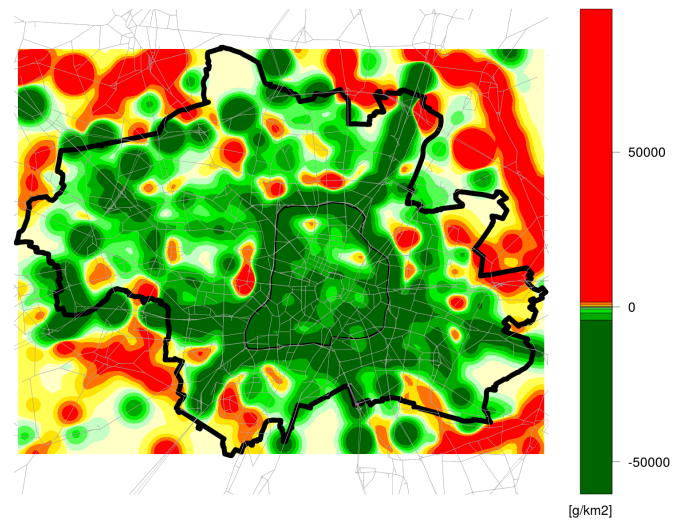

(b) Exposure toll

Figure 9: Absolute changes in $N O_{x}$ emissions. Plots based on spatial averaging for all road segments. Values scaled to a $100 \%$ scenario.

absolute $N O_{x}$ emissions are strongly reduced in the regulated zone, emission levels rise on the middle ring road, as well as on tangential motorways and some arterials. The increase in population exposure from commuter, reverse commuter, and freight traffic might be due to the fact that activity location density is rather high around the middle ring road. In total, the Zone 30 does not reduce $N O_{x}$ emissions $( \pm 0.0 \%)$. The changes of $N O_{x}$ emissions resulting from the exposure toll scheme is shown in Fig. 9(b). As one can notice, the toll reduces emissions almost everywhere in the city area, most importantly along the major corridors, including the middle ring road. However, outside the city area, substantial increases of $N O_{x}$ emissions can be observed. That is, the re-route effect is even more important than for the Zone 30 policy as it pushes travelers out of the city area. In consequence, the exposure toll leads to an overall increase in $N O_{x}$ emissions by $+0.2 \%$.

Both, the re-route effects and the modal shift become also visible in the economic analysis of the two policies shown in Fig. 10. It shows user benefits (and for the exposure toll additionally toll payments) for the different subpopulations. As one can see, the Zone 30 reduces user benefits for all subpopulations. This intuitively makes sense because the transport system is slower than in the base case; this leads to time losses for all car travelers either by switching route or transport mode. The exposure toll, in contrast, yields gains in user benefits for urban travelers. Since these benefits already include the toll payments (as loss in user benefit), it can be followed that for this demand segment congestion relief overcompensates the toll payments. That is, the exposure pricing is implicitly a congestion charge. ${ }^{9}$ When considering the toll payments as transfer payments, the exposure toll has a positive impact on social welfare of the other three subpopulations. That is, toll payments are higher than the direct gains through congestion relief; a redistribution would, however, result in overall benefits for all subpopulations.

\footnotetext{
9 A similar finding was obtained by Kickhöfer and Nagel (2013) for the flat emission toll per $g$, but it seems that the exposure toll even has a stronger positive effect on congestion relief. This might be due to the fact that the exposure toll is correlated more positively with congestion than the emission toll: densely populated areas are implicitly characterized by higher travel demand and therefore pricing exposure influences congestion relief more positively.
} 


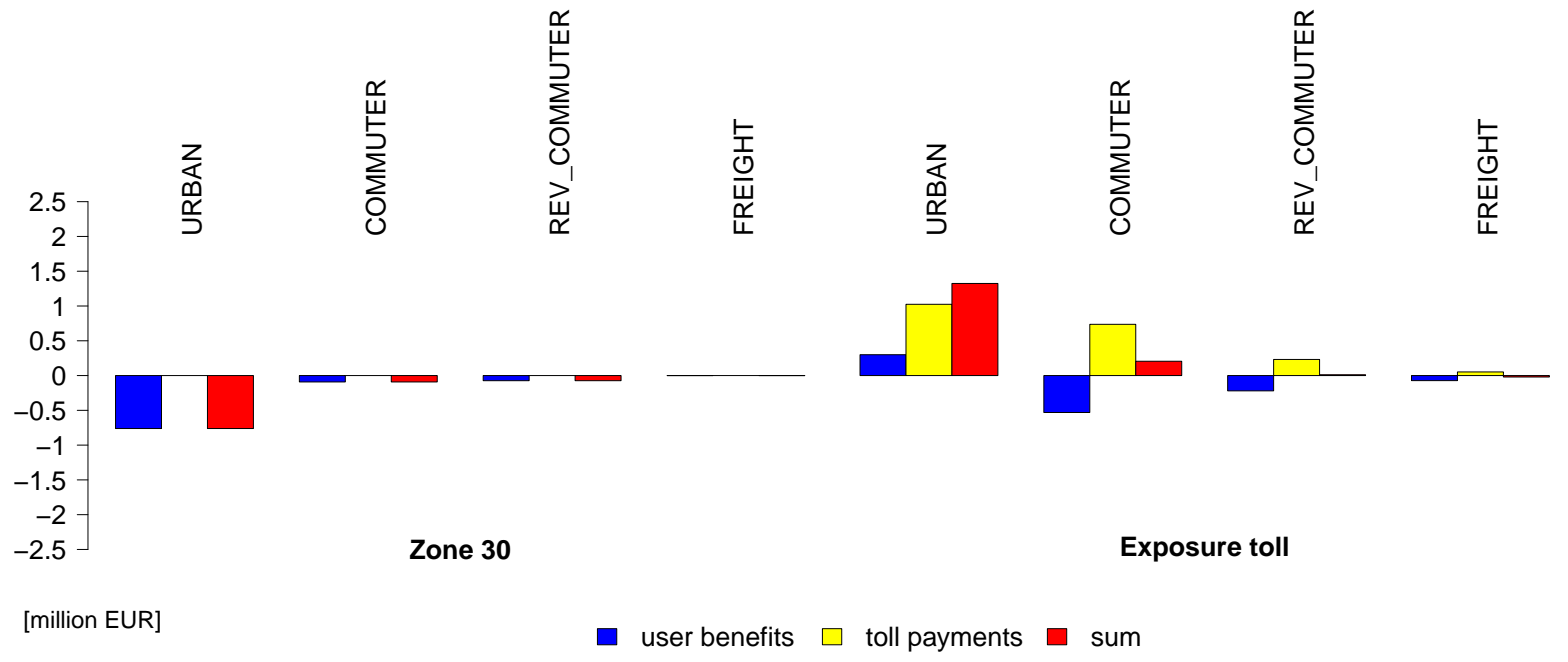

Figure 10: Absolute changes in user benefits by subpopulation.

\section{Conclusion}

This paper proposed a new simplified approach to calculate local air pollution exposure in large-scale urban settings by taking the exposure time derived from the agent-based transport simulation MATSim into consideration. The approach is characterized by little data requirements, reasonable computation times, and open-source compatibility. The main contribution of this paper is therefore that it could prove the feasibility of calculating vehicle-specific, time-dependent exposure tolls iteratively for large-scale scenarios.

For emission modeling, the MATSim-HBEFA tool developed by Hülsmann et al. (2011) was used which calculates warm and cold-start exhaust emissions every time a traveler leaves a road segment. Emission values were monetized using average cost factors from Maibach et al. (2008). Subsequently, the resulting costs were dispersed by a simple Gaussian distribution function applied to discrete cells, and exposure times of affected agents were calculated. Finally, the resulting exposure costs were calculated by scaling the actual exposure times in every cell with the average exposure time of the scenario. Hence, the approach considers activity location density in external cost calculations.

The main advantage of the presented approach is that average cost factors can be used to derive marginal emission costs and map these back to the responsible person. Additionally, there is no need for expensive emission concentration calculations. However, the idea is based on the assumptions that all pollutants are dispersed in the same manner, that the external effects depend on the population density in the same way, and that the average cost factors from the literature are correct for the average exposure time in the scenario.

The implementation was tested in a simple test scenario where the marginal exposure tolls were calculated. It was proven that the implementation influences route choice behavior of agents in the expected way, and that the resulting tolls are influenced by activity location density and activity times of other agents along the route. In order to show the applicability for large-scale scenarios, the approach was then applied to a real-world case 
study of the Munich metropolitan area in Germany. The impacts of the exposure toll on absolute emission levels, exposure cost reductions, and system welfare were then compared to the effects of two policies presented by Kickhöfer and Nagel (2013), who did not account for activity location density in their toll calculations. It was found that the emission toll leads to a strong reduction in emissions, but exposure cost reductions turned out to be much lower than for the proposed exposure toll. For the latter policy, absolute emissions even increase for some demand segments while exposure costs drop. This points out a potential conflict between attempts to optimize exposure (which includes the possibility to drive longer routes with less exposed individuals along them), and attempts to reduce vehicle kilometers traveled in order to mitigate global warming. In that sense, both policies - the emission toll and the exposure toll - can be used as a benchmark for evaluating real-world policies, depending on the objectives. Such benchmarking was performed as a final show case for a speed limitation in the inner city of Munich (Zone 30). It was found that, for urban travelers, the regulated zone seems to be an effective strategy to reduce emission exposure. This is different from the findings in Kickhöfer and Nagel (2013) and is a effect of considering activity location density in the toll calculations. However, in order to reach this cost reduction, the Zone 30 requires a stronger modal shift towards public transit than the exposure toll. Additionally, it yields losses in user benefits while the exposure toll scheme even yields positive welfare effects.

Overall, it can be stated that the emission exposure calculation proposed in this paper improves the evaluation of policies that aim at reducing environmental external costs in urban settings. The eventual goal is to combine the exposure toll with the internalization of other external costs, such as congestion, accidents or noise.

\section{References}

K. W. Appel, C. Chemel, S. J. Rosellea, X. V. Francisc, R.-M. Huc, R. S. Sokhic, S. Raoa, and S. Galmarinid. Examination of the Community Multiscale Air Quality (CMAQ) model performance over the North American and European domains. Atmospheric Environment, 58:142-155, 2012.

R. Arnott, A. de Palma, and R. Lindsey. A structural model of peak-period congestion: A traffic bottleneck with elastic demand. The American Economic Review, 83(1):161-179, 1993. ISSN 00028282. URL http://www.jstor.org/stable/2117502.

N. Cetin, A. Burri, and K. Nagel. A large-scale agent-based traffic microsimulation based on queue model. 2003. Earlier version, with inferior performance values: Transportation Research Board Annual Meeting 2003 paper number 03-4272.

F. Creutzig and D. He. Climate change mitigation and co-benefits of feasible transport demand policies in Beijing. Transportation Research Part D: Transport and Environment, 14(2):120-131, 2009. ISSN 13619209. doi: 10.1016/j.trd.2008.11.007.

T. L. Friesz, D. Bernstein, and N. Kydes. Dynamic congestion pricing in disequilibrium. Networks and Spatial Economics, 4(2):181-202, 2004. doi: 10.1023/B: NETS.0000027772.43771.94.

C. Gawron. An iterative algorithm to determine the dynamic user equilibrium in a traffic simulation model. International Journal of Modern Physics C, 9(3):393-407, 1998. 
K. Geurs and B. van Wee. Backcasting as a tool for sustainable transport policy making: the environmentally sustainable transport study in the Netherlands. European Journal of Transport Infrastructure Research, 4(1):47-69, 2004.

M. Hatzopoulou and E. J. Miller. Linking an activity-based travel demand model with traffic emission and dispersion models: Transport's contribution to air pollution in Toronto. Transportation Research Part D: Transport and Environment, 15(6):315-325, 2010. ISSN 1361-9209. doi: 10.1016/j.trd.2010.03.007.

M. Holland, A. Hunt, F. Hurley, S. Navrud, and P. Watkiss. Methodology paper (volume 1) for service contract for carrying out cost-benefit analysis of air quality related issues, in particular in the clean air for Europe (CAFE) programme. Technical Report 1, AEA Technology Environment, February 2005.

N. Holmes and L. Morawska. A review of dispersion modelling and its application to the dispersion of particles. Atmospheric Enivronment, 40(30):5902-5928, 2006.

F. Hülsmann, R. Gerike, B. Kickhöfer, K. Nagel, and R. Luz. Towards a multi-agent based modeling approach for air pollutants in urban regions. In Proceedings of the Conference on "Luftqualität an Straßen", pages 144-166. Bundesanstalt für Straßenwesen, FGSV Verlag GmbH, 2011. ISBN 978-3-941790-77-3. Also VSP WP 10-15, see www.vsp. tu-berlin.de/publications.

F. Hülsmann, B. Kickhöfer, and R. Gerike. Air pollution hotspots in urban areas - how effective are pricing strategies to comply with the EU limits for $\mathrm{NO}_{2}$ ? In R. Gerike, K. Roller, and F. Hülsmann, editors, Strategies for Sustainable Mobilities: Opportunities and Challenges, pages 105-128. Ashgate Publishing Ltd, 2013. ISBN 978-1-40945489-2.

F. Hurley, A. Hunt, H. Cowie, M. Holland, B. Miller, S. Pye, and P. Watkiss. Methodology paper (volume 2) for service contract for carrying out cost-benefit analysis of air quality related issues, in particular in the clean air for Europe (CAFE) programme. Technical Report 2, AEA Technology Environment, February 2005.

IWW, IFEU, KuP, PÖU, and PTV. Entwicklung eines Verfahrens zur Aufstellung umweltorientierter Fernverkehrskonzepte als Beitrag zur Bundesverkehrswegeplanung. Schlussbericht für Forschungsprojekt FE Nr. 10506001(alt) / 29554001 (neu), Inst. f. Wirtschaftspolitik und Wirtschaftsforschung, U Karlsruhe; Inst. f. Energie- und Umweltforschung GmbH, Heidelberg; Kessel u Parner, Freiburg; Planungsgruppe Ökologie u Umwelt, Hannover; PTVConsult GmbH, Karlsruhe, 1998. Im Auftrag des UBA.

B. Kickhöfer and K. Nagel. Towards high-resolution first-best air pollution tolls. Networks and Spatial Economics, pages 1-24, 2013. doi: 10.1007/s11067-013-9204-8.

B. Kickhöfer, F. Hülsmann, R. Gerike, and K. Nagel. Rising car user costs: comparing aggregated and geo-spatial impacts on travel demand and air pollutant emissions. In T. Vanoutrive and A. Verhetsel, editors, Smart Transport Networks: Decision Making, Sustainability and Market structure, NECTAR Series on Transportation and Communications Networks Research, pages 180-207. Edward Elgar Publishing Ltd, 2013. ISBN 978-1-78254-832-4. 
R. Lindsey and E. T. Verhoef. Congestion modelling. In D. Hensher and K. Button, editors, Handbook of Transport Modelling, volume 1, chapter 21, pages 353-373. Elsevier Science, Oxford, 2000.

M. Maibach, D. Schreyer, D. Sutter, H. van Essen, B. Boon, R. Smokers, A. Schroten, C. Doll, B. Pawlowska, and M. Bak. Handbook on estimation of external costs in the transport sector. Technical report, CE Delft, 2008.

OECD. Cost-benefit analysis and the environment: recent developments. Organisation for Economic Cooperation and Development (OECD), 2006. ISBN 92-64-01004-1. Authored by G. Atkinson, S. Mourato, and others.

I. Parry and K. Small. Does Britain or the United States have the right gasoline tax? The American Economic Review, 95(4):1276-1289, 2005. doi: 10.1257/0002828054825510.

A. Pigou. The Economics of Welfare. MacMillan, New York, 1920.

B. Raney and K. Nagel. An improved framework for large-scale multi-agent simulations of travel behaviour. In P. Rietveld, B. Jourquin, and K. Westin, editors, Towards better performing European Transportation Systems, pages 305-347. Routledge, London, 2006.

K. A. Small and E. T. Verhoef. The economics of urban transportation. Routledge, 2007. ISBN 9780415285148

A. C. Stern, R. W. Boubel, D. B. Turner, and D. L. Fox. Fundamentals of Air Pollution. Academic Press, 2 edition, 1984.

U.S. EPA. The benefits and costs of the clean air act from 1990 to 2020. Technical Report Final Report - Rev. A, U.S. Environmental Protection Agency Office of Air and Radiation, April 2011.

W. Vickrey. Congestion theory and transport investment. The American Economic Review, 59(2):251-260, 1969.

WHO Europe. Air Quality Guidelines Global Update 2005. Technical report, WHO, 2006. 